الآثار الاقتصادية للرى بمياه متباينة الملوحة لبعض محاصيل الحبوب بمحافظة الفيوم

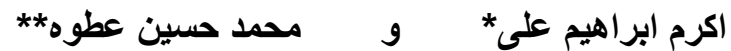

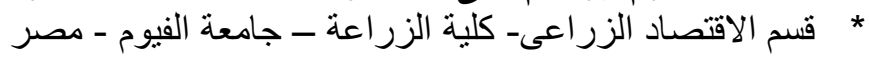

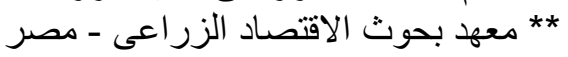

تعتبر مياه الصرف الزر اعى أحد المصـادر التى يمكن إعـادة استخدامها بعد خلطهـا بالميـاه

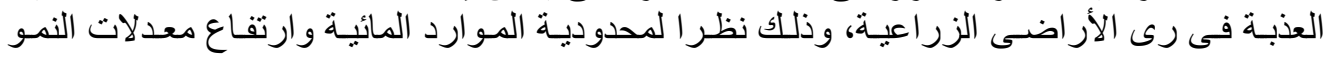

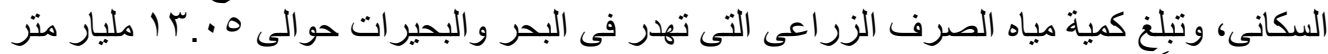

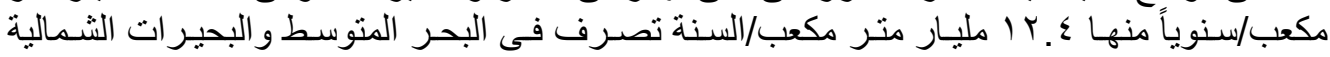

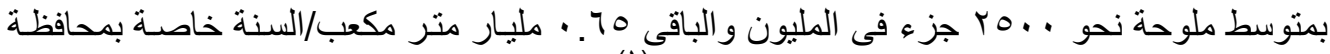

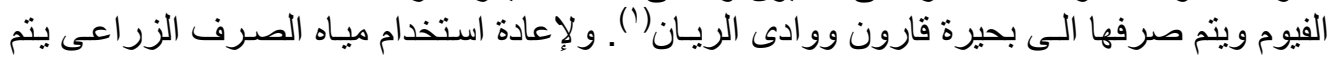

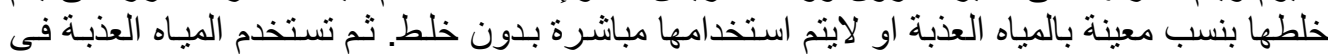

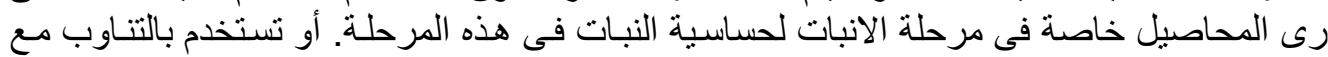

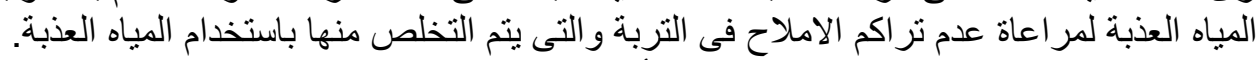

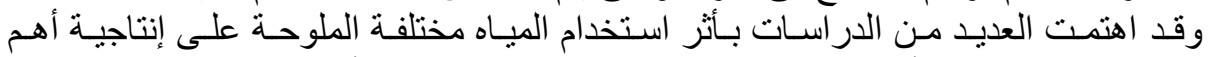

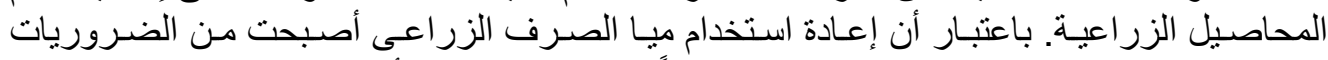

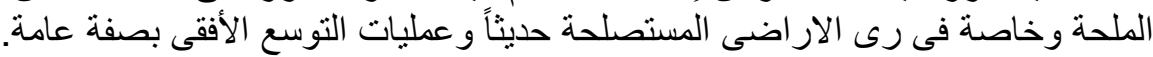

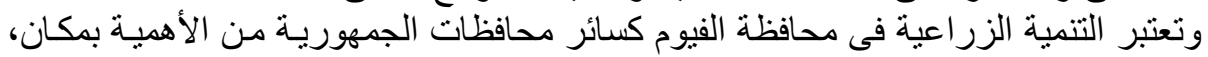
و واصبح تقدم هذه التنمية بمعدلات مرتفعة من الأمور التى تتطلب استثمار الموارد المائية بكفاءة عالية.

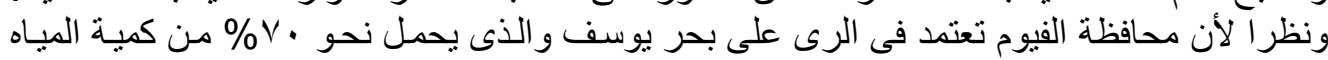

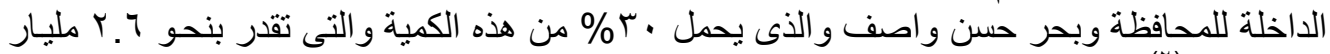

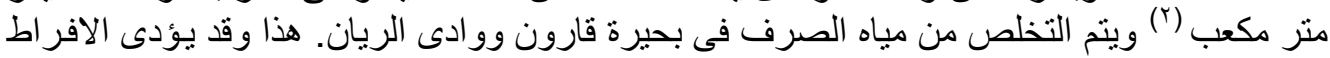

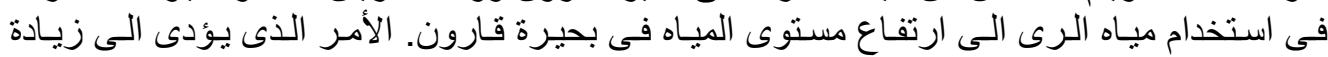

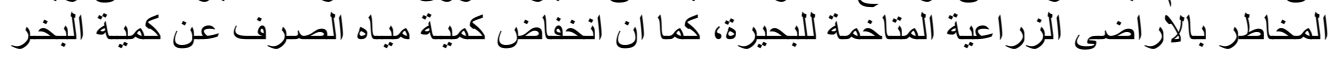

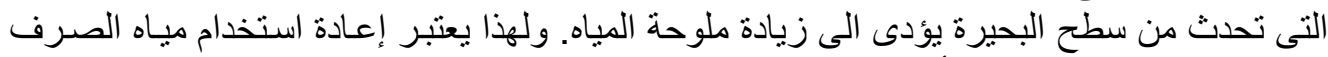

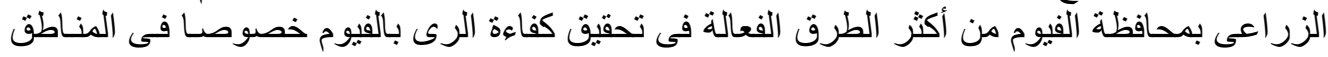

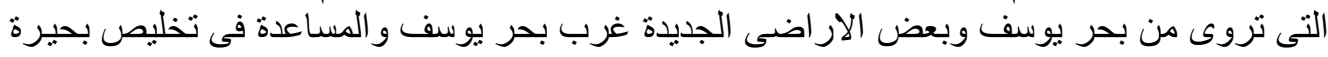

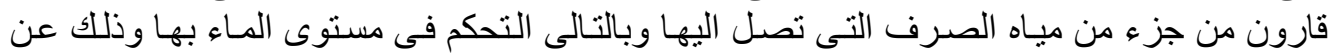

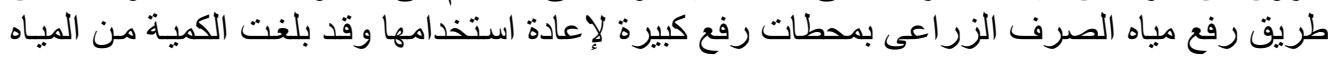

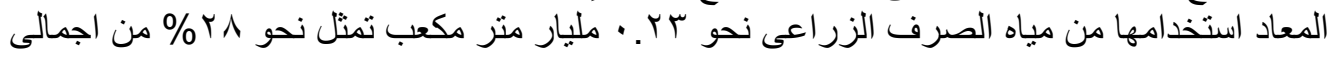

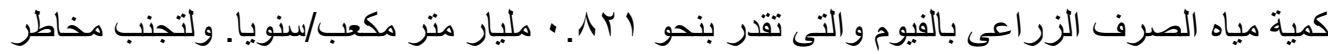

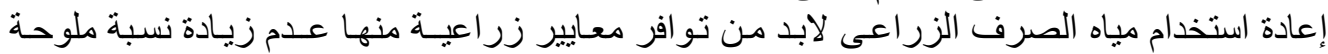

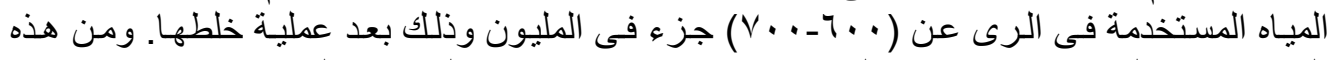

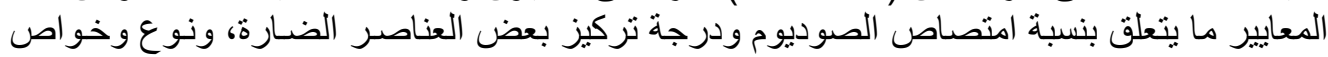

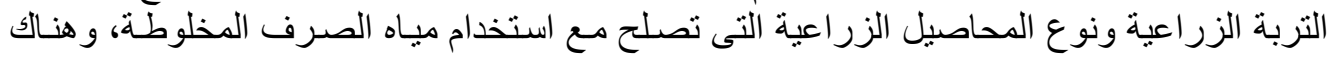

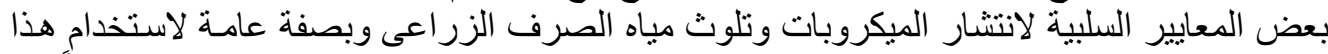

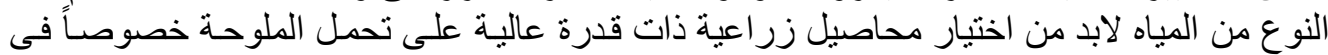

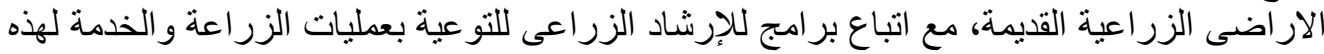

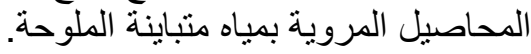

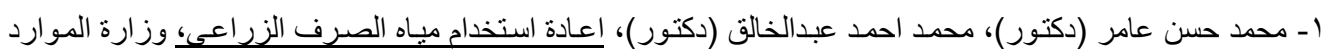

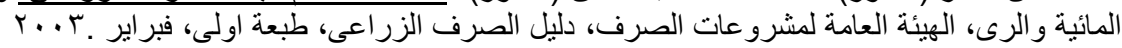

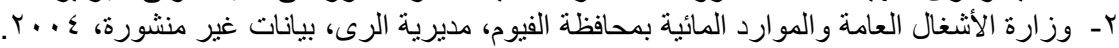

Fayoum J. Agric. Res. \& Dev., Vol. 21, No.1, January, 2007 


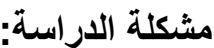

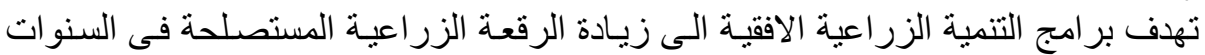

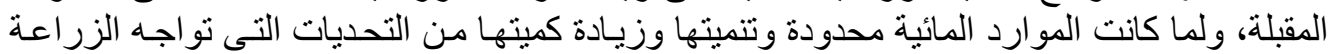

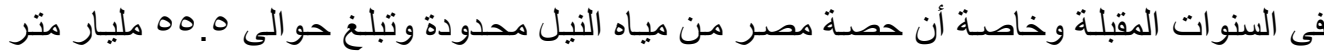

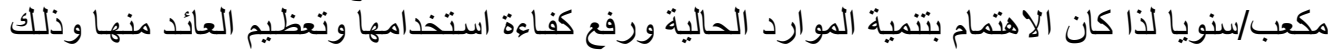

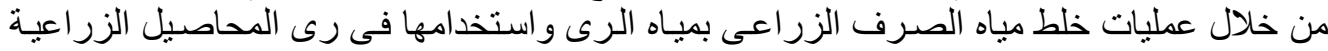

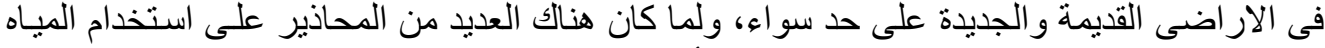

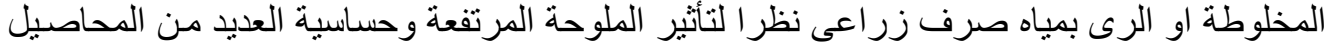

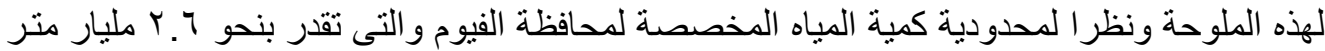

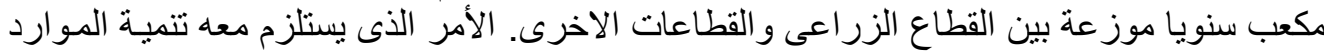

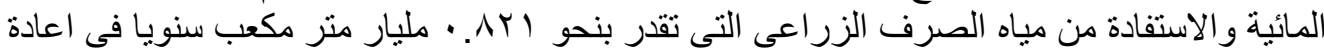

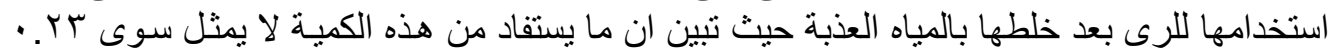

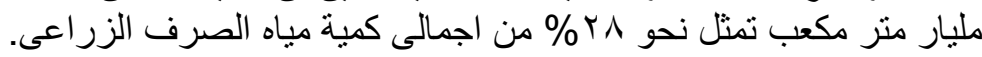

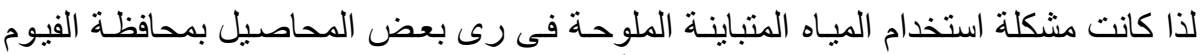

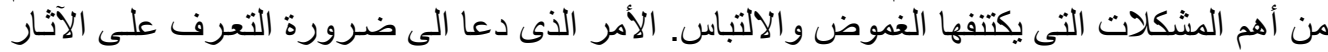

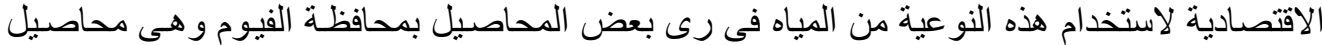

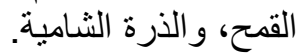

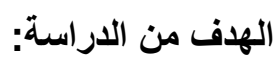

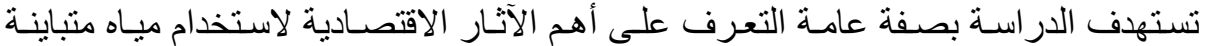

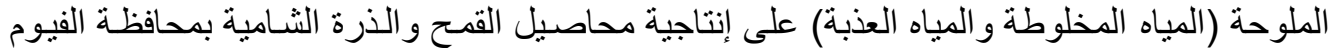

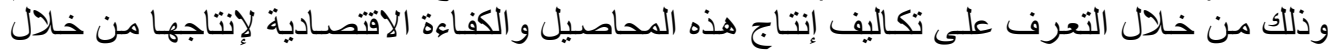

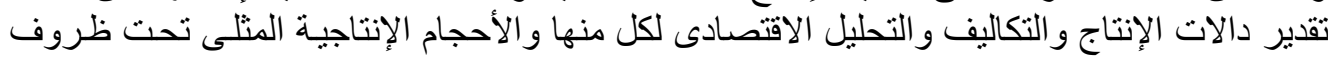
رى هذه المحاصيل بمياه متباينة الملوحة. الطريقة البحثية ومصادر البيانات:

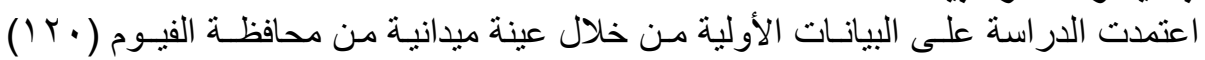

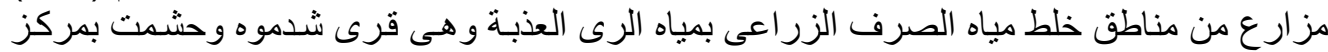

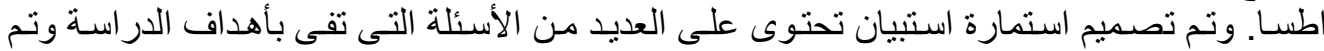

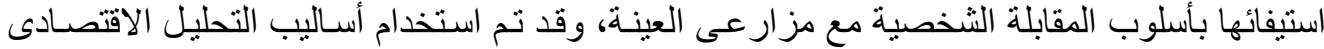
و الاحصائى الوصفى و الكمى للوصول إلى نتائج الدراسة.

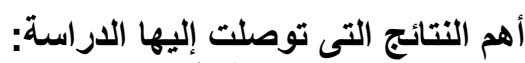
بنود التكاليف الإتتاجية لأهم المحاصيل موضع الإلئ الاراسة:

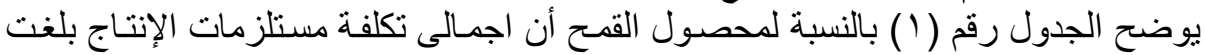

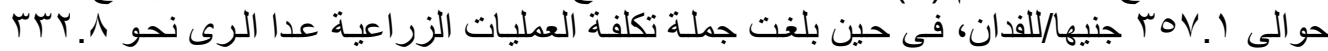

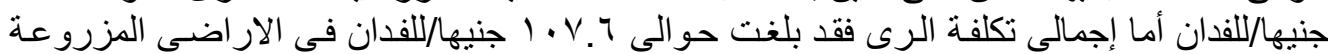

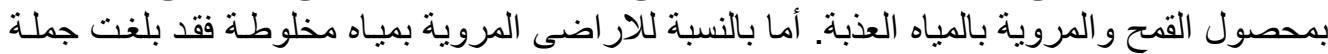

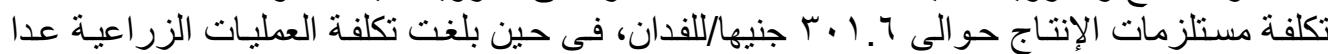

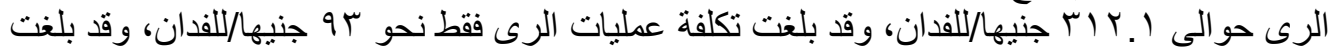

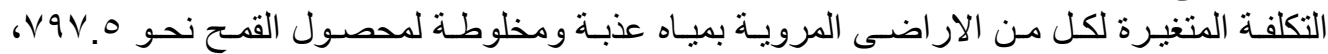

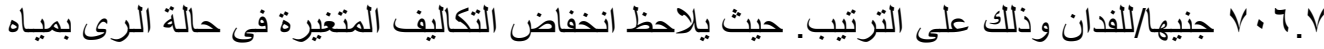

مخلوطة عنها في حالة الرى بمياه عذبة المبة. جدول رقم ( ) : أهم بنود التكاليف المتغيرة للفدان لمحصول القمح وفقا لنوعية المياه المستخدمة فى الرى (القيمة بالجنية)

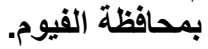

Fayoum J. Agric. Res. \& Dev., Vol. 21, No.1, January, 2007 


\begin{tabular}{|c|c|c|c|}
\hline مياه مخلوطة & مباه عذبه & \multicolumn{2}{|l|}{ نوعية المياه } \\
\hline & & \multicolumn{2}{|c|}{ بنود التكاليف } \\
\hline$V \varepsilon . T$ & $0 \leqslant .0$ & سماد بلدى & \\
\hline A). & $1 \cdot \varepsilon .7$ & 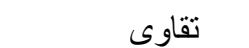 & \\
\hline 110.1 & 149.7 & سماد أزوتي & تكلفة مستلزمات \\
\hline M. & $0 \wedge . \varepsilon$ & سماد فو سفاتي & 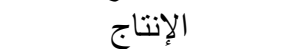 \\
\hline$\Gamma \cdot 1.7$ & rov.l & جملة & \\
\hline 1 Ho.A & $10 \cdot .7$ & العمل الآلى & العمليات الزر اعية عدا \\
\hline IVI.r & IAt.r & 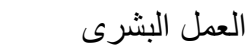 & الرى \\
\hline ril & A.r. & 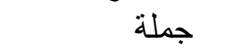 & \\
\hline ov. & $\Lambda \cdot r$ & العمل الآلى & \\
\hline ب." & rV.r & العمل البشرى & عملية الرى \\
\hline 94. & $1 \cdot V .7$ & 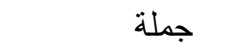 & \\
\hline$V \cdot 7 . V$ & $\vee 9 \vee .0$ & \multicolumn{2}{|c|}{ جملة التكاليف المتغيرة } \\
\hline
\end{tabular}

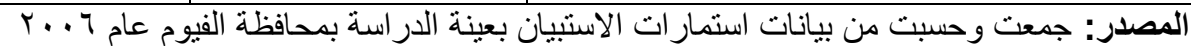

أمـا بالنسبة لمحصول الذرة الثـامية فقد بلغت تكلفة مستلزمات الإنتاج للار اضـى المرويـة

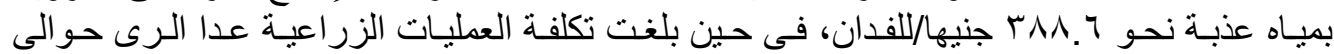

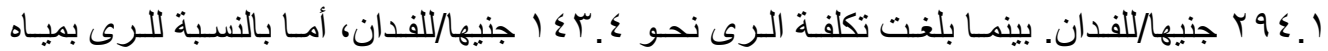

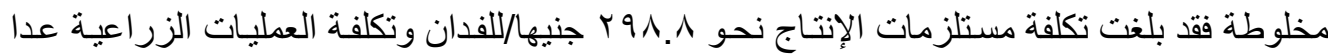

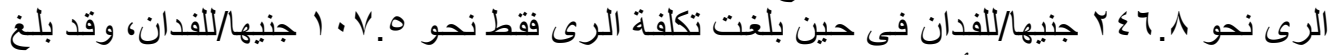

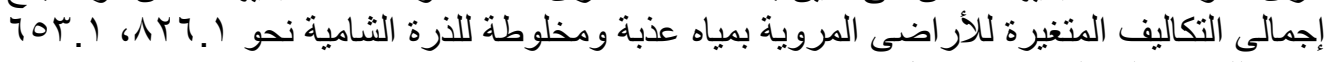

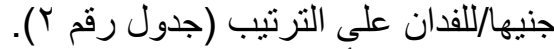

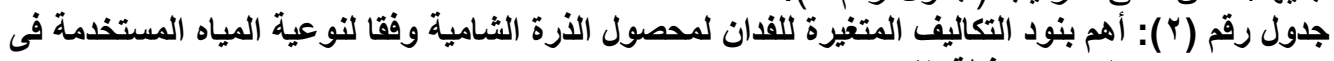

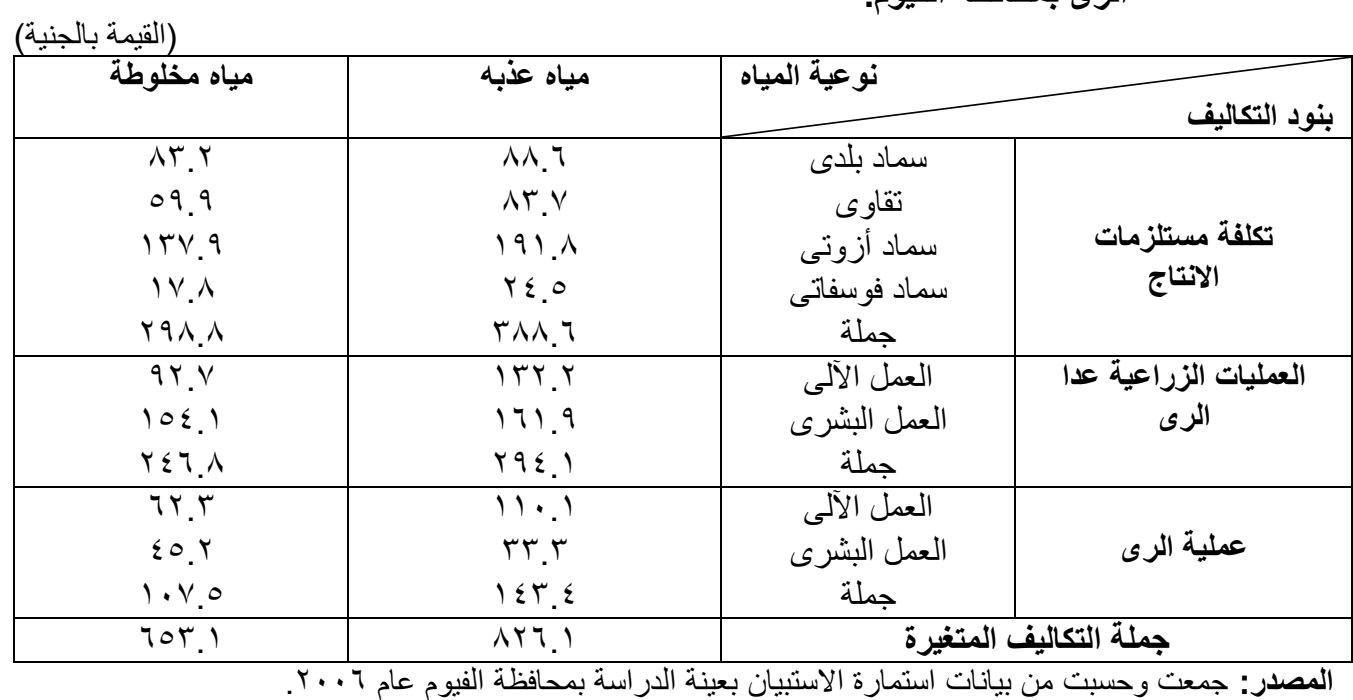

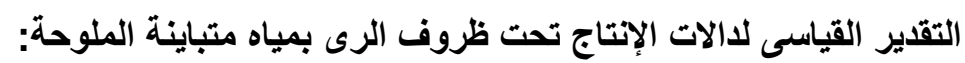

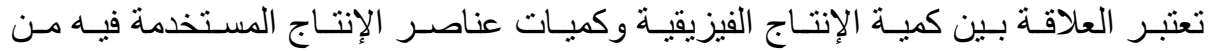

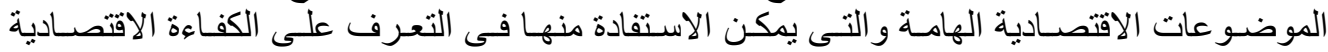
لاستخدام هذه العناصر، وتتوقف كمية الإنتاج الناتجة من تضافر هذه العناصر على الأساليب الإنتاجية

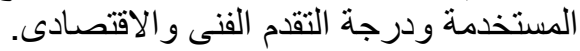

Fayoum J. Agric. Res. \& Dev., Vol. 21, No.1, January, 2007 
rᄉ

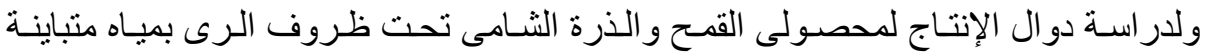

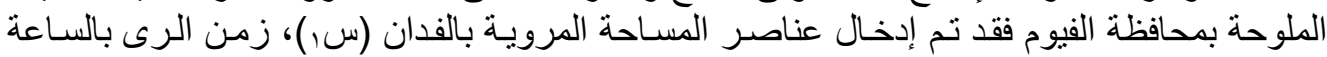

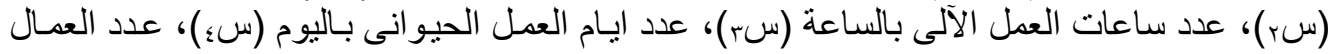

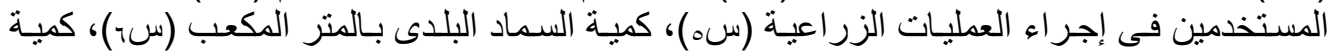

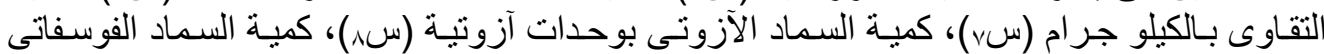

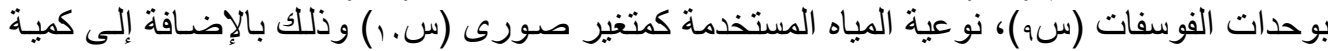

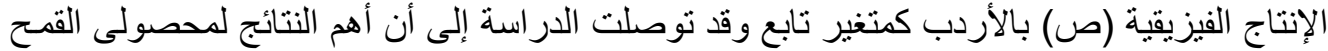

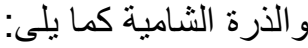

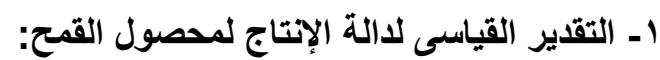

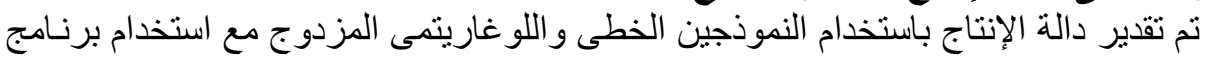

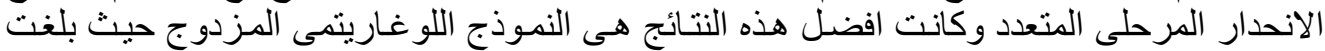

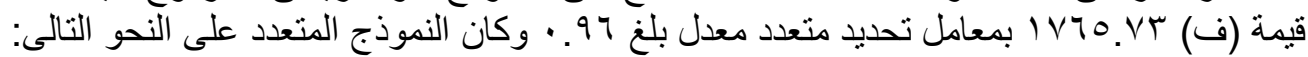

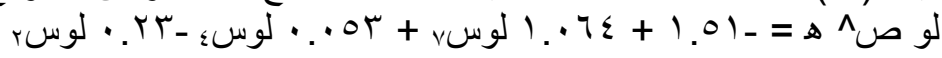
*(r. $\leqslant 7-) \quad * *(0 . V \leq \cdot) \quad * *(1 \leq . \leq V)$

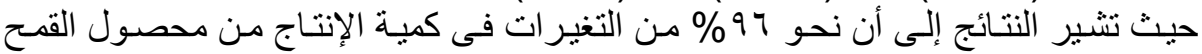

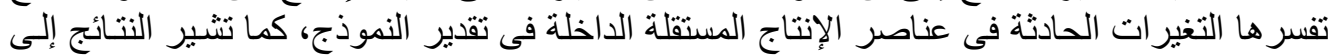

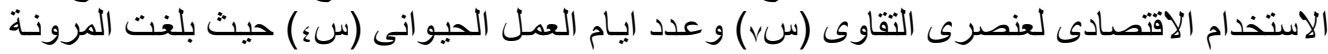

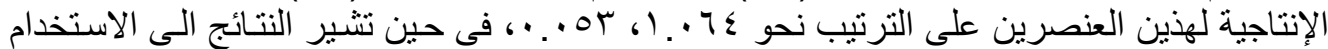
غير الاقتصادى للزمن المستخدم فى الرى بالساعة (سr) حيث تشير إلى استخدام وقت أو زمن أطول

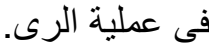

وتثبر نتائج النموذج إلى أن المرونة الإجمالية للنموذج بلغت نحو ANV. • الأمر الذي يثير

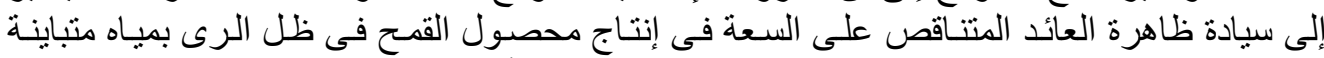

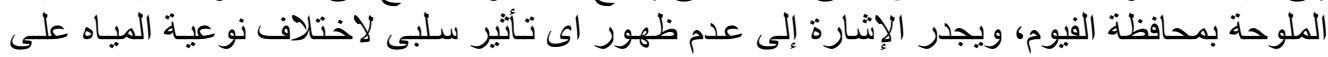
رى محصول القمح.

r- التقدير القياسى لدالة الإنتاج لمحصول الأرة الثامية:

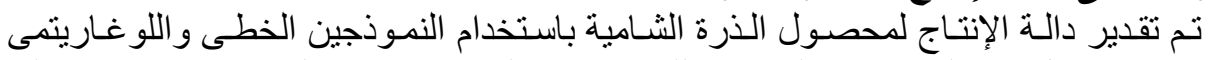

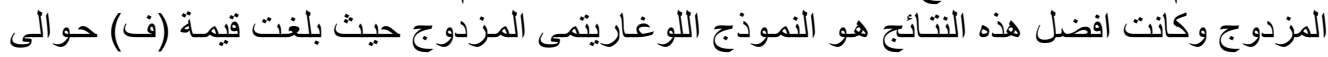

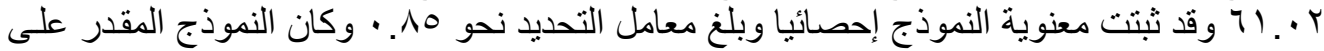

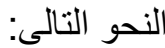

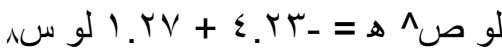
$\left({ }^{* *} . \wedge 1\right)$

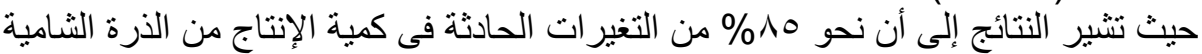

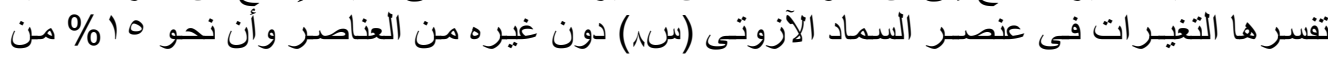

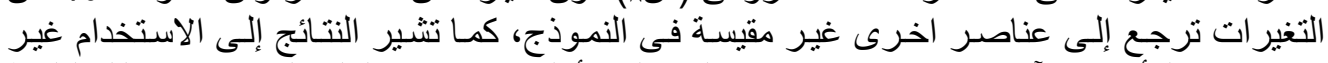

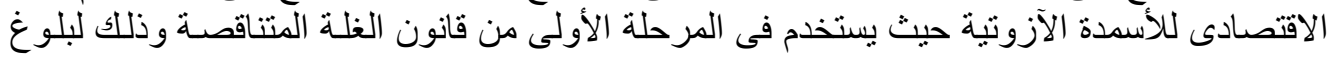

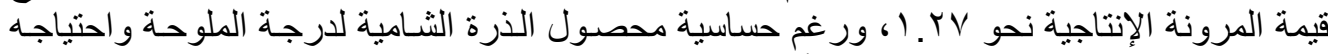

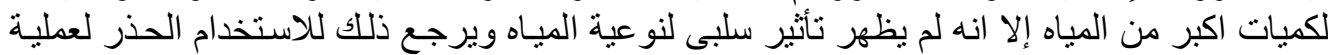
الخلط اى انه يتم عادة الخلط بنسبة ا : : 1 حيث تصبح الملوحة في هذه الحالة ضمن الحدود المسموح

التحليل الاقتصادي لتكاليف الانتاج تحت ظروف الرى بمياه متباينة الملوحة:

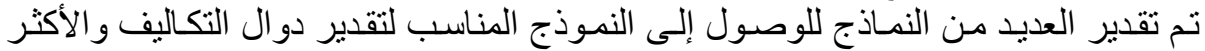

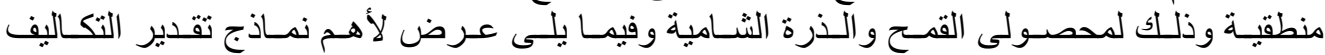

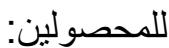

Fayoum J. Agric. Res. \& Dev., Vol. 21, No.1, January, 2007 


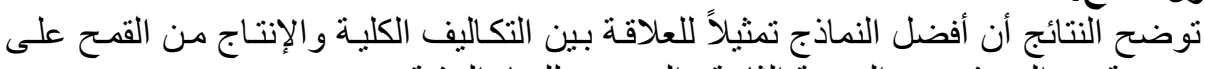

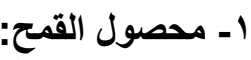

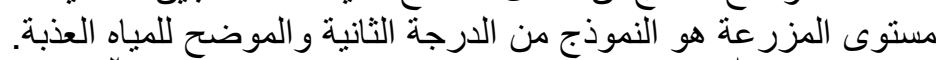

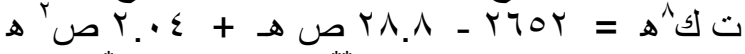

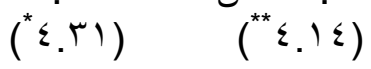

(r79.7) ف

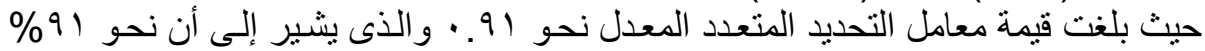

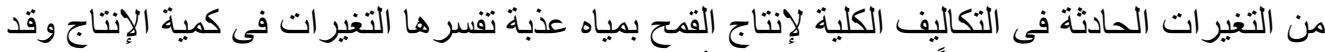

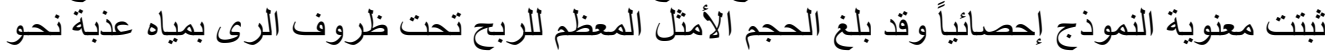

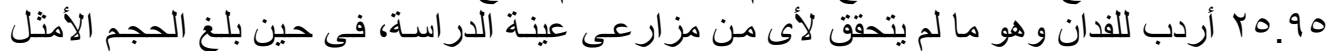

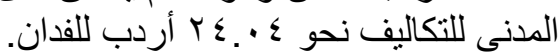
أما فيما يتعلق بالنموذج المقدر للتكاليف الكلية فى حالة الرى بمياه مخلوطة لمحصول القـح

$$
\begin{aligned}
& \text { ت كك }
\end{aligned}
$$

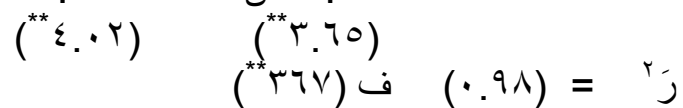

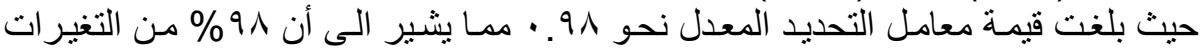

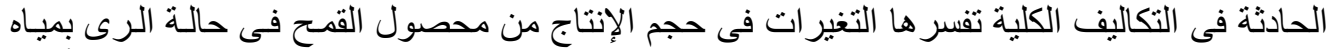

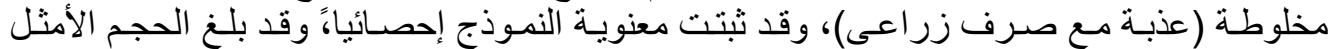

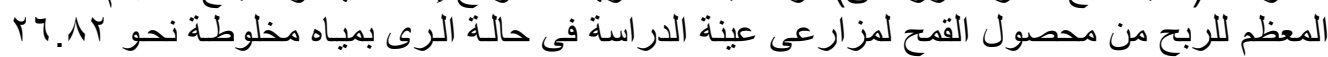

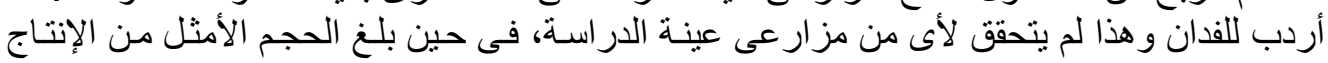

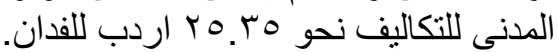

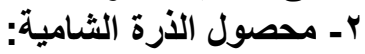

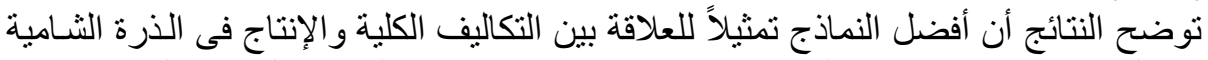

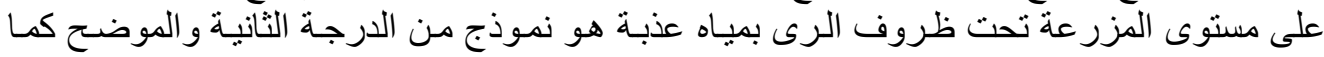

$$
\text { ت ك }
$$

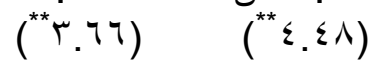

$$
(V \cdot r) \text { ف }(\cdot .9 r)=r
$$

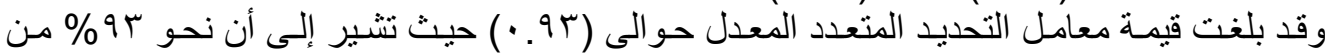

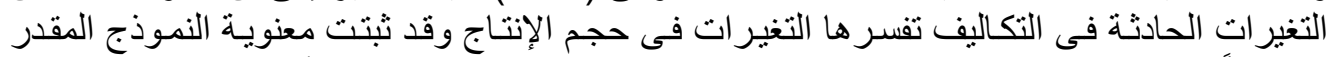

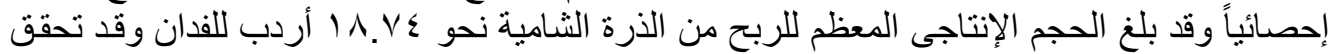

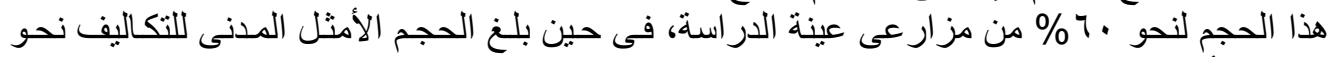

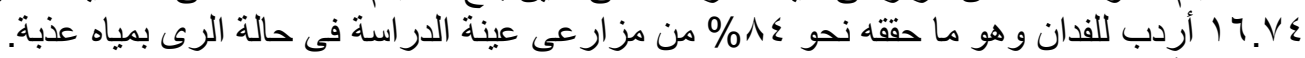

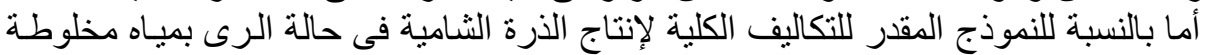

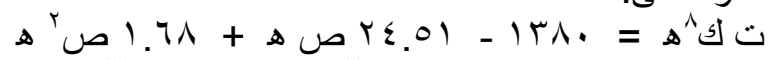

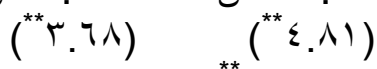

$$
\begin{aligned}
& \text { ( ر }
\end{aligned}
$$

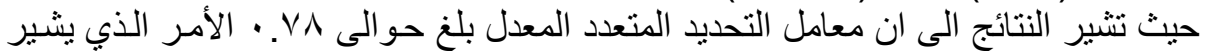

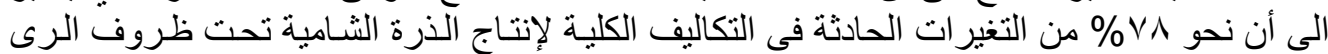

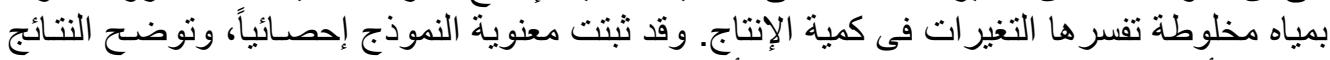

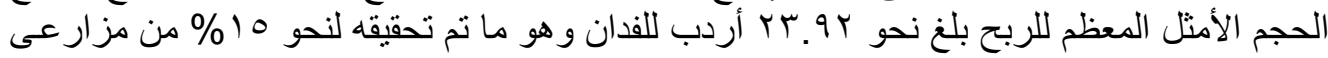

Fayoum J. Agric. Res. \& Dev., Vol. 21, No.1, January, 2007 
$\mu$.

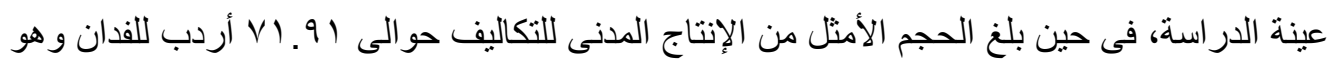

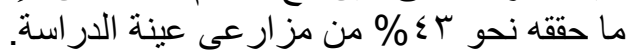

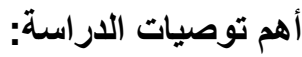

توصي الدر اسة في ضوء ضورة ما سبق من نتائج وللتغلب على الآثار السلبية لاستخدام مياه أكثر ملوحة بمـا يلى:

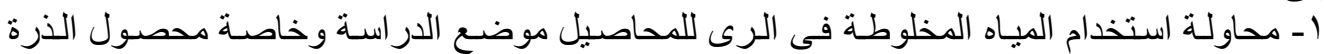
الثـامية بكميـة أكبر ومر اعـاة نسب الخلط ( (: ( ) و الغسيل المتكرر للأر اضـي المرويـة بالميـاه

المخلوطة.

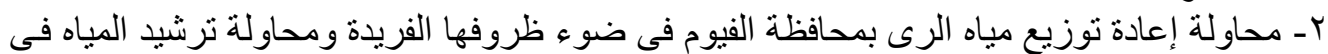

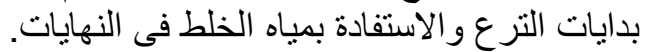

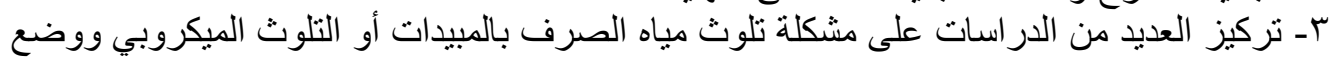

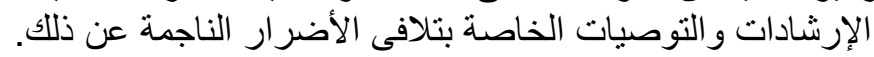

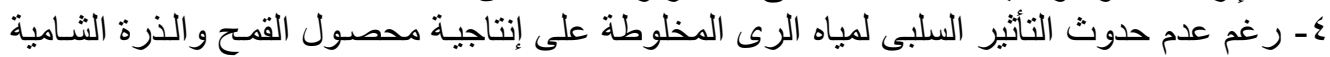

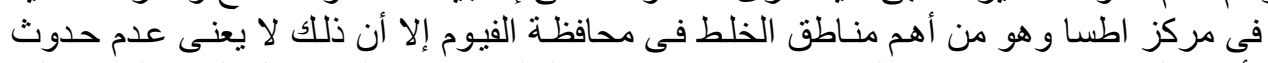

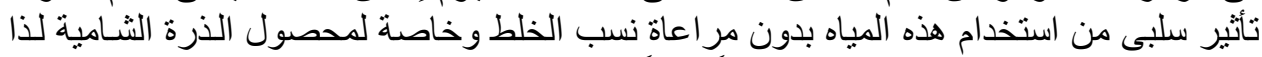

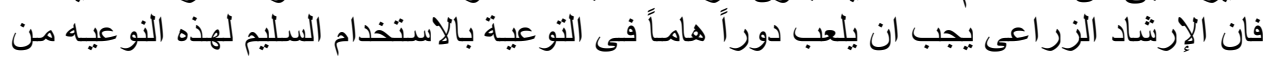

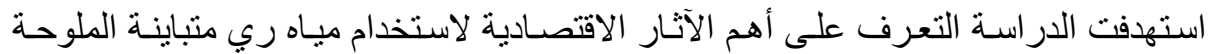

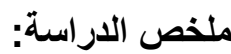

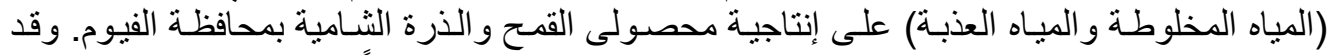

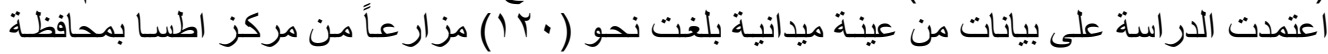

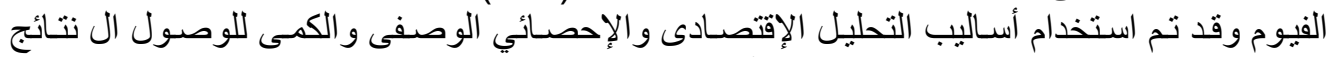
الدراسة، وقد توصلت الدار استة إلى النتائج الآتية:

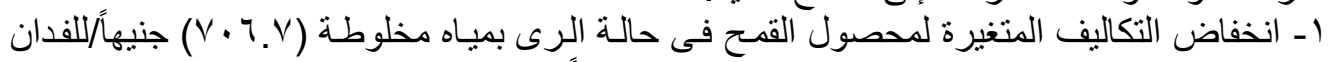

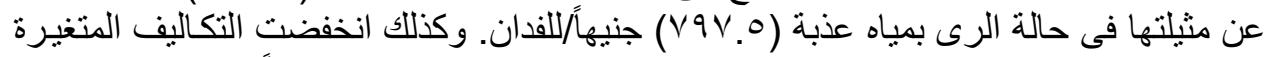

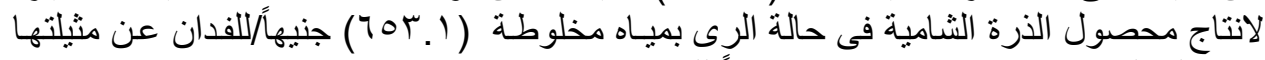

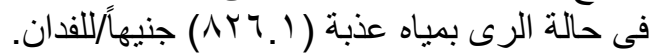

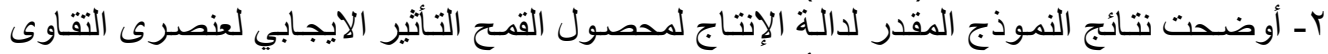

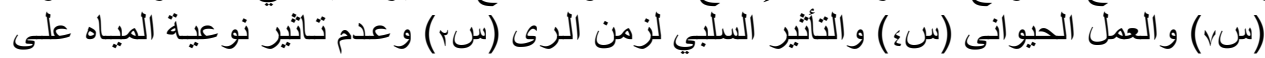

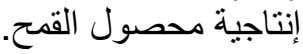

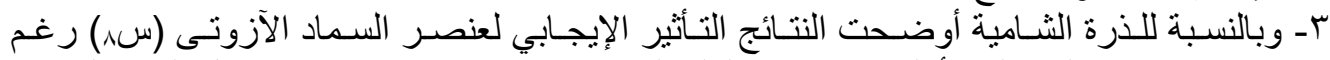

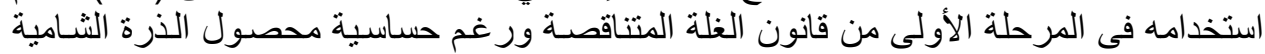

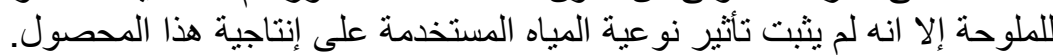

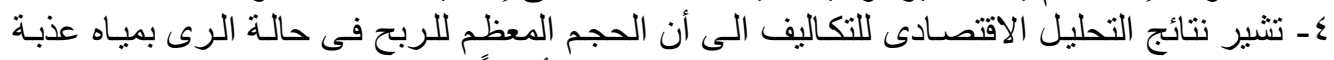

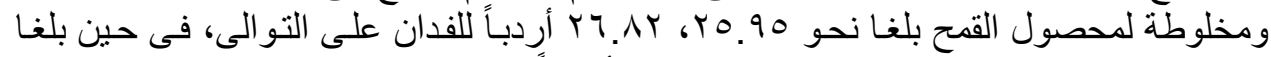

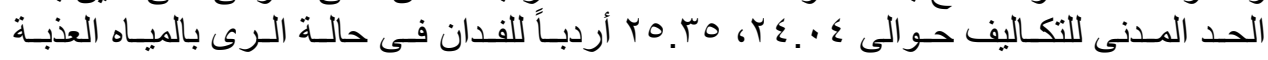

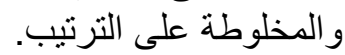

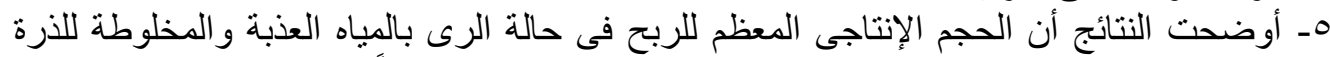

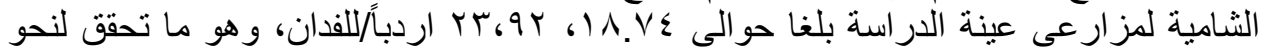

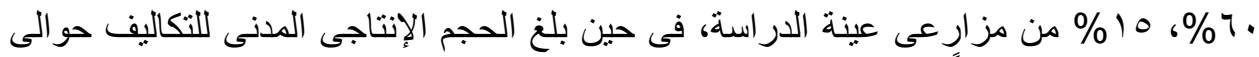

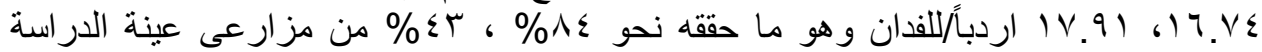

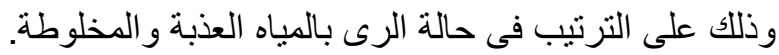

Fayoum J. Agric. Res. \& Dev., Vol. 21, No.1, January, 2007 
M

7ـ أوصت الدر اسة بضرورة اتباع الأسلوب السليم وخلط مياه الصرف الزر اعى بمياه الرى ومر اعـاة

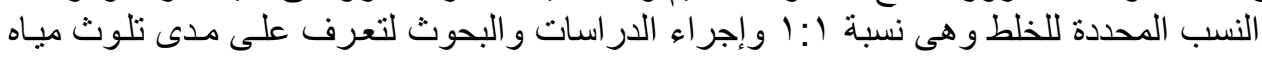

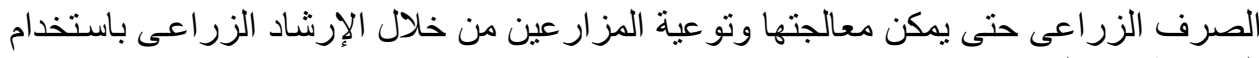

المياه بطرق سليمة.

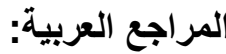

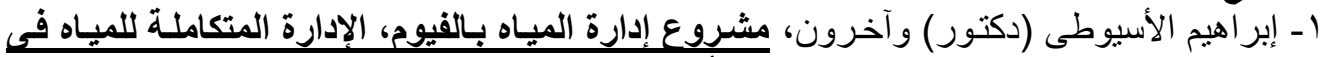

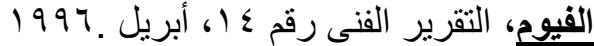

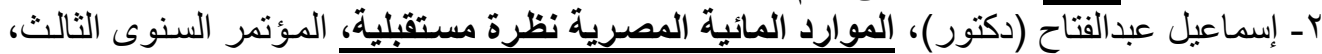

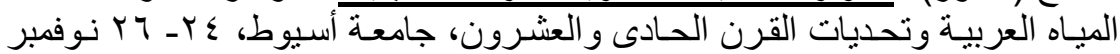

1991 .

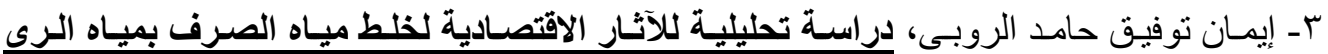

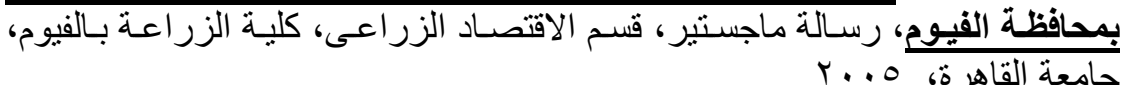

ع- جمال السيد محمد أحمد، اقتصاديات الموارد لمائية وكفاءة الرىى الحقلمى بمحافظة الفيوم، رسالة

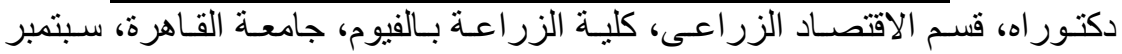

$199 \mathrm{~V}$.

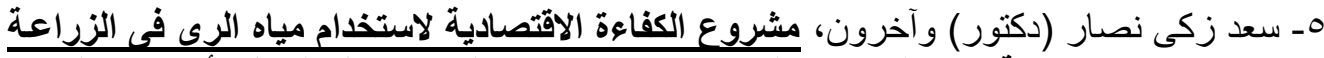

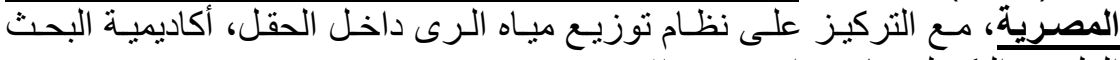

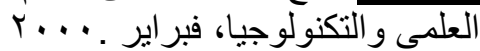

7- سبد صـالح سبد صـلاح (دكتور)، الآثار الاقتصـادية لخلط ميـاه الصـرف بمبـاه الرىى بمحافظة

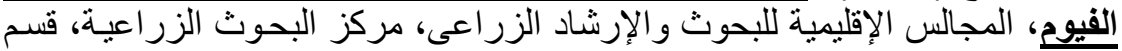

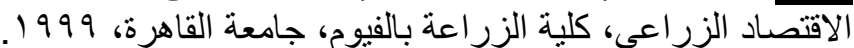

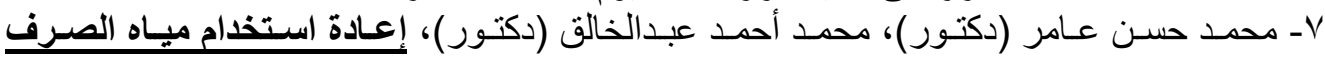

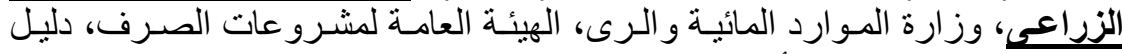

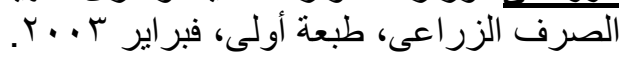

المراجع الأجنبية:

1- Drainage Research: Water Security Project, Technical Report Prepared by the Drainage Research in Stitute Vol. (1), Cairo, Egypt, 1993.

2- FAO: The use of Saline Waters For Crop Production, Land and Water Development Division, Report No. 48, 1992.

\section{THE ECONOMICAL EFFECTS OF IRRIGATION WITH DIFFERENT SALINE WATER ON SOME CEREAL CROPS IN FAYOUM GOVERNORATE}

\section{Akrm I. Ali* and Mohamed H. Atwa** \\ * Faculty of Agriculture - Fayoum University, Egypt \\ ** Agricultural Economics Institute - ARC, Egypt}

\section{ABSTRACT}

The study aimed at recognizing the most important economic effects of using irrigation with different saline water (mixed water and fresh water) on 
the productivity of wheat and corn crops in Fayoum Governorate. The study depended on stratified sample data (120) farmers from Itsa District, Fayoum. The economical, quantative and qualitative analysis were used. The obtained results were found to be as the following:

1- The variable costs of wheat crop reduced with mixed irrigation water (706.7 L.E./feddan), compared to that irrigation with fresh water $(797.5$ L.E./feddan), and for the corn crop, they reduced to 653.1 L.E./feddan with the mixed water compared to the fresh water use (826.1 L.E./feddan).

2- The production function model results for wheat crop revealed the positive effect of seeds $\left(\mathrm{x}_{7}\right)$ and animal work $\left(\mathrm{x}_{4}\right)$, and the negative effect of irrigation time $\left(\mathrm{x}_{2}\right)$, in addition to there was no effect of water type.

3- For the corn crop, the obtained results clarified the positive effect of nitrogen fertilizer $\left(\mathrm{x}_{8}\right)$, although it used in the first stage of the low of diminishing returns. Also, there was no effect of irrigation water type on the corn crop productivity, although its sensitivity to salinity.

4- The costs economical analysis results for wheat crop showed that the optimum size to maximize profit was; 25.95 ardeb/feddan and 26.82 ardeb/feddan for irrigation with fresh water and mixed water, respectively. Whileas, the optimum size to maximize the cost was found to be; 24.04 and 25.35 ardeb/feddan for irrigation with fresh and mixed water, respectively.

5- The obtained results indicated that the optimum size to maximize of production to maximize production for corn crop was; 18.74 and 23.92 ardeb/feddan which was achieved for $60 \%$ and $15 \%$ of the farmers in the study for irrigation with fresh and mixed water, respectively. However, the optimum size to maximize of production to maximize production of costs was; 16.74 and 17.91 ardeb/feddan which was achieved by $84 \%$ and $43 \%$ farmers in the study, respectively.

6- The study recommended that the proper and correct method must be followed and mix the drainage water with fresh water with a ratio of 1:1, in addition to conducting more researches and investigations for treatments of polluted drainage water and use the agricultural extension to guide the farmers. 